\title{
Subjetividad y biopolítica. La pregunta por «la vida»
}

\author{
Subjectivity and Biopolitic. The Question about «Life»
}

\author{
CECILIA CIENFUEGOS MARTÍNEZ*
}

\begin{abstract}
Resumen: Con la modernidad se establece una nueva definición del terreno de lo político. En este artículo se reflexionará en torno a las implicaciones de lo que autores como Michel Foucault o Hannah Arendt han dado en llamar "la entrada de la vida" en la discusión política. De la puesta en común de estos diagnósticos surgen toda una serie de interrogantes con respecto a la posibilidad de la subjetividad contemporánea, que se abordarán a partir de las aportaciones de Judith Butler y sus teorías a propósito de la precariedad, la vulnerabilidad y el reconocimiento.

Palabras clave: biopolítica, sujeto, vida, reconocimiento, precariedad.
\end{abstract}

\begin{abstract}
With modernity a new definition of the political is established. This article will reflect on the implications of what authors such as Michel Foucault or Hannah Arendt have called "the entrance of life" in the political. From the sharing of these insights, a range of questions will emerge about the possibility of contemporary subjectivity. These questions will be approached by using the contributions of Judith Butler and her theories about precariousness, vulnerability and acknowledgment.
\end{abstract}

Keywords: biopolitics, subject, life, acknowledgment, precariousness.

\section{Del poder de muerte al poder de vida: la modernidad según Foucault}

En la modernidad se lleva a cabo todo un proceso de subjetivación del mundo que nos rodea. A cada momento, el ser humano es más consciente de su poder sobre este mundo, hasta el punto de ser capaz de reconocerse como responsable incluso de los cambios meteorológicos, las catástrofes naturales o la expansión epidémica de la muerte. Existe una relación directa con la vida en la medida en que nos sabemos capaces de gestionar los recursos que nos rodean para hacerla más o menos vivible. De esta manera, tal y como apunta Michel Foucault, dado que "el hecho de vivir ya no es un basamento inaccesible que sólo

\footnotetext{
Fecha de recepción: 10/06/2016. Fecha de aceptación: 17/07/2016.
}

* Becaria de postgrado de la Fundación "La Caixa” y estudiante del Máster en Gender Studies (Research) en la Universidad de Utrecht. Antigua estudiante del Máster en Teoría y Crítica de la Cultura, en la Universidad Carlos III de Madrid. Galardonada con el Premio Elisa Pérez Vera, 2014, entregado por el Centro de Estudios de Género de la Universidad Nacional de Educación a Distancia, al trabajo de investigación "Diálogos de Justicia. Reconocimiento, género y precariedad en los debates contemporáneos de justicia", así como con el V Premio de Investigación en Estudios de Género "Ciudad de la Cerámica", 2016, al trabajo: "Sujetos de Violencia. Una aproximación crítica a la violencia sexual como discurso de género". 
emerge de tiempo en tiempo, en el azar de la muerte y su fatalidad, pasa en parte al campo de control del saber y de intervención del poder" ${ }^{1}$. Es decir, la subsistencia ha dejado de ser un problema de primer orden, y la propia dimensión biológica de la vida, que hasta hace relativamente poco resultaba una incógnita, un "regalo" del que poco o nada podía saberse y que nada tenía que ver con la gestión humana, ha pasado a estar en el centro de la preocupación política porque se entiende por primera vez como una cuestión de responsabilidad pública. Cuestiones de estricta naturaleza biopolítica como la protección ante el dolor, el derecho al aborto (y por lo tanto a la gestión del propio cuerpo por parte de las mujeres), o el mismo derecho a la propia muerte, son cuestiones protagonistas de la discusión éticopolítica contemporánea, que ponen sobre la mesa interrogantes políticos fundamentales sobre la autonomía, la libertad o la soberanía.

Con esta subordinación de lo biológico a la gestión y producción humanas, la vida entra en la polis, y esto supone automáticamente toda una serie de cambios en nuestra estructura de comprensión de lo político. En palabras de Foucault: "Durante milenios, el hombre siguió siendo lo que era para Aristóteles: un animal viviente y además capaz de una existencia política; el hombre moderno es un animal en cuya política está puesta en entredicho su vida de ser viviente"2. Y lo que ocurre es que, con este cambio fundamental, Foucault diagnostica una transformación en la lógica de legitimación del poder, que está directamente relacionada con las mejoras técnicas modernas que han hecho cada vez más accesible la conservación de la vida. Así, en un desarrollo paralelo al auge del capitalismo, el poder deja de estar ocupado simplemente por los "sujetos de derecho" y pasa a centrar su atención en los "seres vivos", de manera que su misma naturaleza se ve transformada. El poder soberano característico de los Estados-nación, que basaba su legitimación y máxima autoridad en su potencia de muerte, da paso a una nueva forma de poder que parte ahora de la propia vida, y toma como fuente de soberanía y legitimidad el hacer esta vida posible.

Según Foucault, este cambio de paradigma se establece y refuerza, a partir de los siglos XVII y XVIII, según dos polos: por un lado, con la focalización en las disciplinas del cuerpo, basadas en la necesidad de construir cuerpos cada vez más capaces, sanos y útiles para la producción capitalista, pero al mismo tiempo dóciles y sumisos; y por otro lado, con toda una serie de controles y regulaciones de la población, centrados en un cuerpo humano como especie cuyos procesos han de ser medidos y reajustados de acuerdo a los intereses de un determinado Estado-nación ${ }^{3}$. El cambio fundamental atañe por tanto a qué se considera dentro del terreno de lo político. Este cambio, además, tiene que ver con una importancia creciente de la norma, en contraposición al sistema jurídico de la ley; y, con esta transformación fundamental, el poder pasa de estar armado y ser un poder basado en la amenaza de muerte, a convertirse en un poder regulador, basado en dispositivos de definición y corrección constantes según los criterios establecidos de valor y utilidad. La "vida" se convierte en el objetivo político por excelencia, y con su "invasión” adquiere el poder su acceso inmediato al cuerpo como terreno político.

1 Foucault, M., Historia de la sexualidad 1. La voluntad de saber. Madrid, Siglo XXI, 2009, p. 151.

2 Ibid., p. 152.

3 Es en la intersección de estos dos ejes donde se coloca el sexo; y es por esto, según Foucault, por lo que el sexo como tal se articula como un reto para el juego político contemporáneo, que lo convierte en el blanco de todo tipo de vigilancias, regulaciones y sistemas de control. 
El problema es que lo que se plantea es una reivindicación tremendamente incomprensible para el sistema jurídico clásico; esto es: la reivindicación de un "«derecho a la vida», al cuerpo, a la salud, a la felicidad, a la satisfacción de las necesidades; el «derecho», más allá de todas las opresiones o «alienaciones», a encontrar lo que uno es y todo lo que uno puede ser". Es decir, cuando Foucault habla de "derecho a la vida", no habla de "cualquier vida" como nuda vida, sino de una vida que permita el desarrollo del sujeto en los términos en los que éste quiera desarrollarse. A lo que nos enfrentamos aquí, por lo tanto, es a una pregunta fundamental por la subjetividad, y ésta es: ¿en qué términos es un sujeto posible? Lo que nos lleva quizá a plantear una pregunta anterior, como la siguiente: ¿de qué hablamos cuando hablamos de "vida"? Pero para abordar estas preguntas, es necesario primero reflexionar sobre qué significa la entrada de la vida en la esfera de la política, y cuál es la relación entre ambas categorías. En este sentido, las aportaciones han sido muchas, pero considero imprescindible reparar en las reflexiones de la filósofa alemana Hannah Arendt, que en muchos sentidos terminó por desarrollar una teoría paralela a la planteada por el filósofo francés, aunque de conclusiones opuestas.

\section{Hannah Arendt: La política de la acción y la negación del cuerpo}

En su influyente libro La condición humana, Arendt realiza un desalentador y descarnado diagnóstico del mundo contemporáneo, cuya conclusión es el reconocimiento del ascenso del homo laborans al centro de la escena política, y con él la politización de la nuda vida, que traerá consigo la condena de la capacidad de acción política. Es decir, con la entrada de la vida en la polis se sentencia para Arendt el eclipse de la política, y con él se configura la entrada en la modernidad. Arendt desarrolla su análisis en torno a tres categorías fundamentales: la labor, el trabajo y la acción. De estos tres conceptos, la acción (praxis) sobresale como lo imprescindible de la condición humana, lo único que expresa su genuina esencia. Lo característico de la acción, para Arendt, es que sólo puede darse allí donde las necesidades más inmediatas están cubiertas, y su función es la de establecer una suerte de comienzo libre en el tiempo, un comienzo de algo radicalmente nuevo. Una de las características más incómodas de la acción, no obstante, es que sus resultados son tan irrevocables como impredecibles. Debido a esto, existe la tentación constante de encontrar un sustituto a la acción, y así "escapar de los asuntos humanos para adentrarse en la solidez de la quietud y el orden"5. No obstante, para Arendt, el problema de los tiempos modernos es que se caracterizan por el eclipse de la acción, que es tan sólo concebible en algunas excepciones revolucionarias -siempre y cuando partan de una radical autonomía con respecto a aspectos sociales y aspiren a instaurar una absoluta novedad histórica en términos estrictamente políticos-.

Lo característico de nuestra época, por lo tanto, no es su dimensión fabril o técnica, sino que hemos retornado a esos ciclos elementales de la supervivencia que representa la labor y nos hemos reducido así a una existencia cíclica, en virtud de la cual vamos constantemente dando vueltas sin saber muy bien por qué, en una actividad incesante que no configura mundo. La política misma ha terminado convertida en una suerte de labor cuya función es la

4 Foucault, M., Historia de la sexualidad 1. La voluntad de saber. Madrid, Siglo XXI, 2009, p. 154.

5 Arendt, H., La condición humana. Barcelona, Paidós, 2005, p. 243. 
de satisfacer las necesidades contingentes, establecidas en términos de felicidad -que recibe también el nombre de bienestar- y cuya estructura esencial es la del consumo. Esto hace que la acción en sentido estricto sea algo casi olvidado, que puede irrumpir desde luego de manera inesperada en alguna revolución, pero que no tiene nada que ver con el espíritu de los tiempos.

Es decir, según Arendt, a partir de la modernidad se habría dado el surgimiento de lo que ella llama la "esfera de lo social", que sin ser ya una esfera estrictamente privada, como sí lo es el oikos aristotélico, traslada las lógicas de este último al espacio público, y esto es algo tremendamente nocivo para la propia construcción de lo político. Con esta ascensión, la frontera entre lo público y lo privado se diluye, y el cometido fundamental de la gestión de lo común pasa a centrarse en la administración de la vida contingente: de sus procesos y urgencias cotidianas. Y lo que ocurre es que, para Arendt, si la polis habría de ser el espacio de la acción libre de los hombres, esta libertad sólo puede estar garantizada en la medida en la que sus contingencias y necesidades inmediatas no intervienen en la discusión pública, pues éstas no hacen sino corromper toda posibilidad de acción verdaderamente política. De esta manera, la división entre lo público y lo privado se erige en Arendt como una condición fundamental para la construcción política, y es por esto por lo que resultaría implanteable, desde su punto de vista, una noción como la de "bio-política" - a la que ella en todo caso nunca hará referencia-. En el momento en el que la vida se inmiscuye en las cuestiones de la polis, toda posibilidad política queda condenada a un falso ejercicio de libertad.

Esta incompatibilidad radical llama desde luego la atención, por lo que cabe pararse a reflexionar sobre cuáles son las propiedades o características que convierten a la vida en una cuestión tan problemática para la noción arendtiana de política. Me permitiré destacar en este sentido dos aspectos que resultarán en adelante fundamentales en lo que refiere a la reflexión que aquí se plantea, a propósito de la posibilidad de una "subjetividad biopolítica".

En primer lugar, habría que preguntarse por cuáles son las características del espacio público en Arendt. En este sentido, destaca como la primera de sus condiciones la configuración de un espacio de "igualdad entre los diferentes", que parta de reconocer la pluralidad constitutiva de la condición humana, pero que no vulnere en todo caso el principio de igualdad entre pares - una condición necesariamente construida y que sólo puede darse fuera del hogar y la familia, esfera que Arendt reconoce como el centro natural de la desigualdad-. En Arendt son por tanto indispensables los otros, en la medida en que "la acción, a diferencia de la fabricación, nunca es posible en aislamiento [...]. La fabricación está rodeada y en constante contacto con el mundo; la acción y el discurso lo están con la trama de los actos y palabras de otros hombres"6. Esta pluralidad, no obstante, apunta Arendt, no tiene nada que ver con la alteridad. Ésta, de hecho, distorsiona por completo el espacio público: supone un elemento perturbador que de alguna manera desvirtúa todas las relaciones humanas y hace imposible el discurso y la escucha en igualdad de condiciones. En este sentido cabe pensar, desde luego, en una tensión directamente relacionada con la entrada de lo femenino en el espacio público: una entrada cuyo efecto inmediato parece ser el de una suerte de automática "privatización" del mundo.

$6 \quad$ Ibid., p. 216. 
Frente a esta característica de lo público, la actividad de la labor consiste precisamente en aquella "en la que el hombre no está junto con el mundo ni con los demás, sino solo con su cuerpo, frente a la desnuda necesidad de mantenerse vivo" ". De esta manera, la segunda condición imprescindible del espacio público, que tiene que ver con la posibilidad de libertad, nos lleva a ese ya citado rechazo de la necesidad y contingencia de la vida humana, que ha de ser gestionada en el espacio privado y bajo el resguardo de la institución de la familia. Es más, Arendt se remite a la esfera de la familia para poner de manifiesto que la inclusión de la vulnerabilidad del cuerpo contingente lleva directamente a la lógica de la dominación, pues donde no hay iguales, y allí donde el hombre actúa movido por la llamada a la conservación del propio cuerpo, aparece la violencia: "Debido a que todos los seres humanos están sujetos a la necesidad, tienen derecho a ejercer la violencia sobre otros; la violencia es el acto prepolítico de liberarse de la necesidad para la libertad del mundo"8. Es decir, en última instancia, la familia se constituye en el discurso arendtiano como ese espacio prepolítico -o antipolítico, a veces-, donde la dominación está legitimada en la medida en que sólo a partir de la misma puede trascenderse la inmediata vulnerabilidad de la propia vida, necesitada y dependiente, para saltar a un mundo político donde estas necesidades queden al margen. Pero para que estas necesidades puedan ser puestas a un lado han de ser satisfechas en última instancia por otro tipo de "sujetos", por los que no hay posibilidad -o necesidadde preguntarse (tradicionalmente encarnados, huelga de nuevo insitir, por las mujeres).

Parece por tanto claro que, según la concepción arendtiana de lo político, lo que molesta profundamente de la entrada de la vida en la esfera pública es su relación con un elemento especialmente incomodo para ésta: la condición corporal del ser humano. El cuerpo, en esta definición de lo político, no es más que aquello que nos aleja de una anhelada "igualdad": en primer lugar, porque instituye una diferencia fundamental e infranqueable entre (al menos) dos tipos de individuos diferentes - uno de ellos, además, definido por su radical corporalidad, su completa y sistemática dependencia de su condición de cuerpo: las mujeres-; y en segundo lugar, porque el cuerpo nos remite constantemente a esa indigencia primera de nuestra existencia, pues somos seres encarnados y por lo tanto vulnerables, dependientes, atados a las exigencias del mundo de la vida. El problema es que, si la concepción tradicional de sujeto da toda preeminencia a la conciencia, el cuerpo es típicamente negado; mientras que la mente se instituye como transparente, lo que ocurre con el cuerpo es que representa una total opacidad. Tal y como plantea David Le Breton en su libro Antropología del cuerpo y modernidad, nada es más misterioso para el hombre que el espesor de su propio cuerpo; y sin embargo, paradójicamente, sólo podemos vivir a partir de él: es la primera instancia a partir de la cual accedemos al mundo.

Lo que quizá debería llamar aquí la atención, por lo tanto, es cómo han podido mantenerse estas dos esferas separadas durante tanto tiempo. Y lo que ocurre es que hemos aprendido a dibujar lo político como el espacio de la emancipación en la medida en que es capaz de mantenerse esencialmente separado del mundo de la vida, por lo que éste tiene de contingente y desigual. Sin embargo, tal y como plantea Carmen González Marín: "la sombra del biopoder nos fuerza a adoptar una perspectiva nueva: carece de sentido hacer

7 Ibid., p. 237.

8 Ibid., p. 57. 
político lo personal, porque lo personal ya es una construcción biopolítica" ${ }^{\prime \prime}$. Es decir, con este "cambio a la biopolítica", el centro del ejercicio del poder pasa a localizarse en la administración de la vida, lo cual implica un cambio en los mecanismos de este mismo poder. Un "bio-poder" que por vez primera ve legitimado su ejercicio ante el único objetivo de conservar la vida pasa a operar en el espacio público; no obstante, lo que cabría entonces preguntarse es si lo determinante es un cambio hacia una lógica biopolítica, o si al contrario ésta siempre estuvo allí, y quizá lo nuevo es la instauración de un poder que construye su legitimidad directamente a partir de ella. Quizá lo que ocurre es que la política no puede abstraerse de la vida si no es mediante un ejercicio de exclusión consustancialmente violenta -como aquel que se lleva a cabo para la construcción de la división entre el espacio público y el privado-. Quizá por tanto no sea la vida lo problemático, sino la negación de la misma, pues condena al olvido toda una esfera de la condición humana que, lejos de ser anterior o contraria a la política, se constituye como el requisito indispensable de la misma.

\section{Política desde el cuerpo: ¿qué significa poner la vida en el centro?}

Así las cosas, si la vida es de alguna manera inseparable de la política, esta "entrada de la vida en la polis" ha de poder entenderse desde alguna otra perspectiva. En este punto, cabe de nuevo recuperar a Foucault, quien realiza un diagnóstico paralelo al de Arendt pero de sentido contrario, dado que, mientras que en Arendt el ascenso de lo social es siempre negativo, pues implica el olvido de la política, en Foucault se trata más bien de reivindicar la política para lo corporal. Es decir, cuando Foucault hace estos análisis en torno a cómo se desarrolla la política moderna como producción de verdad sobre los cuerpos, lo que está reivindicando es que cuando se hace política ha de hacerse desde el cuerpo, desde lo material y lo viviente. Aboga por tanto por la recuperación de la capacidad política de lo puramente vivo, desde la inmediatez de la carne. Pero ¿qué significa esto para la pregunta por el sujeto?

Hablar del sujeto en términos de biopolítica implica pensar que aquello que se subjetiviza es siempre un cuerpo, un cuerpo que está vivo y que es por lo tanto vulnerable. Este punto de partida significa, como hemos visto, una transformación interesante en la manera de abordar el campo de lo político. Pero no sólo eso: a partir de esta transformación la propia noción de sujeto experimenta un cambio fundamental. Partir del cuerpo implica partir de una noción ampliada de interdependencia, pues, tal y como plantea Judith Butler: "Cada cuerpo se define por las relaciones que hacen su vida y acción posibles". Es decir, no se trata de pensar en una serie de sujetos unidos en la esfera pública por sus necesidades individuales, sino de que la propia dependencia de nuestros cuerpos niega la sola posibilidad de un sujeto solipsista y autorreferente. Lo cierto es que no podemos hablar de un cuerpo sin saber qué es lo que sostiene a ese cuerpo. De esta manera, el cuerpo se desvela menos como una entidad que como un sistema de relaciones (entre las que han de contarse toda una serie condiciones materiales o ambientales que permiten su existencia, pero también de dispositivos de control

9 González Marín, C., «Introducción. Biopolítica y género», Cuadernos Kóre. Revista de historia del pensamiento de género (Madrid), Vol. 1, No 4, Primavera/verano 2011, p. 12. 
que hacen a ese cuerpo reconocible por otros). El pensar al sujeto desde el cuerpo permite así poner de manifiesto que no existe acción posible sin el apoyo necesario, pues no puede pensarse en el cuerpo como algo singular o autorreferente.

Pero pensar desde la biopolítica, por otro lado, significa también pensar que lo subjetivo es siempre una construcción dictada por una determinada dinámica de poder, y que por lo tanto nos remite a la pregunta sobre quiénes son los individuos capaces de reclamar reconocimiento y quiénes en cambio quedan fuera de esta posibilidad por no formar parte de los marcos de inteligibilidad impuestos por un régimen de verdad determinado. Es decir, no puede pensarse en un sujeto prediscursivo, anterior a la norma, pues esta norma define previamente la viabilidad o inteligibilidad de un sujeto dado; es decir, decide por anticipado quién puede llegar a ser un sujeto y quién no. La idea de qué es una construcción social tiene que ver por tanto con una construcción de verdad desde cierto sistema de poder, y en este sentido es relevante señalar algo en lo que repara la filósofa norteamericana Judith Butler y que va a constituir un punto de partida fundamental para cualquier reflexión bio-política: y es que la producción implica siempre no solamente la creación de aquello que se quiere producir sino también la generación de residuos, de afueras constituyentes.

Para Butler, que en este sentido es profundamente foucaultiana, el sujeto no es algo dado, sino una construcción dependiente de discursos del poder y del saber. Así, la paradoja es que "si los términos del poder definen quién puede ser un sujeto, quién está cualificado como sujeto reconocido, en política o ante la ley, entonces el sujeto no es una precondición de la política, sino un efecto diferencial del poder"10. Esta afirmación supone, entre otras cosas, una terrible dificultad para las reivindicaciones por el reconocimiento. Y es que lo que ocurre es que "pensamos en los sujetos como el tipo de seres que piden reconocimiento ante la ley o ante la vida política, pero quizás el asunto más importante es cómo los términos de reconocimiento -y aquí podemos incluir una gran cantidad de normas sexuales y de génerocondicionan por anticipado quién será considerado como sujeto y quién no"11. Y es quizá la pregunta por lo abyecto, aquello que habita en los límites de lo reconocible y que no puede reconocerse como "vida", la que constituye el interrogante clave, que nos habla también, de alguna manera, de la condición artificial y construida de aquello que se ha producido como una construcción supuestamente anterior al poder.

Y lo que ocurre es que, de esta manera, la misma categoría del reconocimiento se desvela insuficiente, dado que nos enfrentamos a fenómenos que cuestionan su posibilidad. A partir de aquí, lo que Judith Butler se plantea es un reto interesante: el de construir una nueva ontología corporal que parta de la conciencia de la absoluta vulnerabilidad que nos define como seres humanos. Es decir, que parta de nuestra condición de cuerpos necesitados, que dependen en todo momento de lo que hay fuera de sí mismos para sostenerse. En este sentido, en su libro Marcos de guerra. Las vidas lloradas, Butler parte del problema ontológico planteado por la pregunta ¿qué es una vida?, que desde su punto de vista nos deriva a un problema epistemológico que se configura por su parte bajo la pregunta ¿qué es lo reconocible como vida? Ambas cuestiones, finalmente, devendrán en un problema de responsabilidad

10 Butler, J., «Performatividad, Precariedad y Políticas Sexuales», AIBR. Revista de Antropología Iberoamericana (Madrid), Vol. 4, nº 3, septiembre-diciembre 2009, p. 324.

11 Ibid., p. 325. 
ética, según el cual hemos de preguntarnos por ¿qué hay que reconocer? -teniendo en cuenta que este reconocimiento está ligado a la concesión de una serie de prerrogativas sociales, tales como la protección ante la violencia o la adjudicación de derechos- ${ }^{12}$.

Ante esta primera pregunta ontológica, Butler apunta que "el «ser» de una vida está constituido por unos medios selectivos, por lo que no podemos referirnos a ese «ser» fuera de las operaciones del poder, sino que debemos hacer más precisos los mecanismos específicos del poder a través de los cuales se produce la vida"13. Es decir, la vida, como la noción de sujeto, es siempre una producción diferencial del poder, y esto significa que no todas las vidas cuentan lo mismo. Para ser capaz de registrar esta situación, por lo tanto, Butler plantea la necesidad de partir de una nueva ontología corporal, que implique repensar cuestiones como "la precariedad, la vulnerabilidad, la dañabilidad, la interdependencia, la exposición, la persistencia corporal, el deseo, el trabajo y las reivindicaciones respecto al lenguaje y a la pertenencia social"14. Lo que hay que tener en cuenta, desde la perspectiva butleriana, es que en la medida en que somos seres "expropiados por la presencia del otro", cuya autonomía es siempre dependiente de esta existencia externa, lo que nos constituye políticamente es precisamente "la vulnerabilidad social de nuestros cuerpos"15.

No obstante, la característica principal de esta común vulnerabilidad es que no se encuentra repartida en términos de igualdad. Es decir, si bien estamos condenados a vivir como seres humanos una existencia precaria, esta precariedad se encuentra repartida de manera diferencial, y es aquí donde radica para ella el punto de partida de toda reflexión política que aspire a transformar verdaderamente la sociedad. En este sentido, Butler establece una diferencia entre el concepto de «precariedad» (precariousness) y el de «precaridad» (precarity). Con «precariedad» (precariousness) Butler hace referencia a esa condición compartida de vulnerabilidad intrínseca a todo ser vivo, en la medida en la que somos completamente dependientes de nuestra interacción con los otros para garantizar la propia supervivencia. No obstante, tal y como se ha señalado, el problema es que esta precariedad se encuentra distribuida de manera diferenciada entre cada uno de los individuos y grupos sociales -ya sea por cuestiones de género, de raza, de identidad sexual, de nacionalidad, etc.-. Propone por lo tanto la reflexión sobre el concepto de «precaridad» (precarity) que, frente a la universalidad de la noción de precariedad, hace referencia a una "condición políticamente inducida" que marca las posiciones imposibles de vida.

Esta condición políticamente inducida de la «precaridad» es en buena medida producto ya no sólo de la falta de reconocimiento sino de la imposibilidad de inteligibilidad dentro de los marcos de reconocimiento establecidos. Pasamos por lo tanto a un problema de carácter epistemológico, pues lo que ocurre es que estos marcos normativos de lo cognoscible, dentro de los cuales se aprehende una vida como tal, operan de manera diferencial estableciendo jerarquías entre aquellos cuyas vidas merecen ser protegidas y aquellos cuyas vidas resultarán invisibles y no merecedoras de duelo. En este punto es importante, por lo tanto, tener en cuenta que "esta distribución diferencial de precariedad es, a la vez, una cuestión material y perceptual, puesto que aquellos cuyas vidas no se «consideran» susceptibles de ser lloradas,

12 Butler, J., Marcos de guerra. Las vidas lloradas, Madrid, Espasa Libros, 2010, p. 16.

13 Ibid., p. 14.

14 Ibid., p. 15.

15 Butler, J., Vida precaria. El poder del duelo y la violencia. Buenos Aires, Paidós, 2006, p. 46. 
y, por ende, de ser valiosas, están hechos para soportar la carga del hambre, del infraempleo, de la desemancipación jurídica y de la exposición diferencial a la violencia y a la muerte"16. En este sentido, Butler llama la atención sobre el hecho de que esta vulnerabilidad común que nos constituye como seres sociales, lejos de devenir simplemente en la necesidad del reconocimiento mutuo, establece en primera instancia unas estructuras de dominación determinadas, que se hacen cargo de la delimitación de lo reconocible para justificar las dinámicas de poder establecidas. Es decir, la primera dinámica que implica la distribución diferenciada de la precariedad es la de la instauración de una serie de discursos que aspiran a dar "una explicación específica" -que posteriormente será naturalizada y concebida en términos ontológicos- que justifique la existencia "de poblaciones marcadas, de vidas que no son del todo vidas, que están modeladas como «destructibles» $\mathrm{y}$ «no merecedoras de ser lloradas»"17.

La responsabilidad ética impuesta por la definición de esta nueva ontología corporal pasa por lo tanto por reconocer esta condición diferenciada y en último término injusta de la aprehensión de la precariedad, que finalmente dibuja un panorama político repleto de sujetos invisibles y de vidas invivibles. El asumir la precariedad como punto de partida, por lo tanto, implicaría el recuperar la noción de vulnerabilidad como un supuesto compartido en igualdad de condiciones y asumir las necesidades que ésta implica de cara a la construcción de políticas sociales que centren su atención en garantizar las condiciones de vida necesarias para que una vida sea vivible. Supone, por lo tanto, un cambio radical de enfoque que aspira a poner en el centro de la discusión la pregunta de qué es una vida que merezca la pena ser vivida, para supeditar el desarrollo de toda teoría ética o política posterior a esta pregunta fundamental.

\section{Bibliografía}

Arendt, Hannah (2005): La condición humana, Paidós, Barcelona.

Butler, Judith (2006): Vida precaria. El poder del duelo y la violencia, Paidós, Buenos Aires. Butler, Judith (2009): «Performatividad, Precariedad y Políticas Sexuales», AIBR. Revista de Antropología Iberoamericana, Vol. 4, n 3, septiembre-diciembre 2009, Madrid.

Butler, Judith (2010): Marcos de guerra. Las vidas lloradas, Espasa Libros, Madrid.

Foucault, Michel (2009): Historia de la sexualidad 1. La voluntad de saber. Siglo XXI, Madrid.

González Marín, Carmen (2011): «Introducción. Biopolítica y género», Cuadernos Kóre. Revista de historia del pensamiento de género, Vol. 1, № 4, Primavera/verano 2011, Madrid.

16 Butler, J., Marcos de guerra. Las vidas lloradas, Madrid, Espasa Libros, 2010, p. 45.

17 Ibid., pp. 53-54. 
